\title{
PREVALÊNCIA DE PIROSE EM PELOTAS, RS, BRASIL: estudo de base populacional
}

\author{
Farid NADER ${ }^{1}$, Juvenal Soares Dias da COSTA ${ }^{2}$, \\ Gisele Alsina NADER ${ }^{3}$ e Gledis Lisiane Correa Luz MOTTA ${ }^{3}$
}

RESUMO - Racional - Desconhece-se a prevalência da doença do refluxo gastroesofágico no Brasil e em países latino-americanos, bem como de seu marcador mais específico: a pirose. O conhecimento desse parâmetro epidemiológico é importante pela influência eventual da enfermidade na qualidade de vida, bem como o risco de complicações. Objetivo - Estudar a prevalência de pirose na população adulta na cidade de Pelotas, RS. Delineamento - Estudo transversal de base populacional. Pacientes $\boldsymbol{e}$ Métodos - Foram incluídos indivíduos a partir de 20 anos, residentes na zona urbana da cidade. Utilizou-se amostragem por conglomerados em 40 setores censitários do Instituto Brasileiro de Geografia e Estatística. Questionários padronizados e précodificados foram aplicados aos participantes. Realizou-se controle de qualidade aplicando-se questionários simplificados em $10 \%$ das pessoas incluídas na amostra. A codificação e a entrada de dados através do programa Epi-Info foram realizadas duas vezes, com o intuito de diminuir os erros de consistência. A análise dos dados foi realizada através do programa SPSS, onde foram feitas comparações entre a prevalência de pirose em relação às variáveis demográficas através das razões de prevalências, intervalos de confiança a 95\% e do teste do qui-quadrado. Resultados - Entre as 1.263 pessoas incluídas no estudo, encontraramse taxas de prevalências de pirose de $48,2 \%, 32,2 \%$ e $18,2 \%$ para o último ano, mês e semana, respectivamente. Todas as formas do desfecho pirose estavam associadas com o sexo feminino, de maneira estatisticamente significativa. Contudo, não foi encontrada associação com relação à idade. Conclusões - As elevadas prevalências encontradas neste estudo devem chamar a atenção para assistência médica a estes indivíduos, particularmente ao fato de que estão expostos a eventuais complicações da doença do refluxo gastroesofágico.

DESCRITORES - Pirose, epidemiologia.

\section{INTRODUÇÃO}

A doença do refluxo gastroesofágico (DRGE) é das afecções mais freqüentes na prática médica, sendo a pirose um marcador que apresenta especificidade e valor preditivo positivo para o diagnóstico dessa condição de aproximadamente $100 \%{ }^{(9)}$.

A DRGE têm impacto grande na qualidade de vida dos pacientes, da mesma forma que a úlcera duodenal, a hipertensão arterial, a insuficiência cardíaca congestiva, a angina e mesmo a menopausa ${ }^{(4)}$. Por outro lado, as complicações da enfermidade, particularmente o potencial maligno do esôfago de Barrett, destacam a importância da caracterização e do tratamento da doença.
Estudos epidemiológicos têm evidenciado aumento, nas duas últimas décadas, da prevalência da pirose. É possível que mudanças nos hábitos alimentares, fatores ambientais, o aumento do uso do álcool, aspectos culturais e raciais possam estar relacionados com esse aumento. Assim, dados publicados no Canadá, $4 \%$ dos participantes referiram pirose diária, 30\% pirose semanal. Na Finlândia, 10,3\% referiram pirose diária e $30 \%$ pirose semanal. A ocorrência de pirose anual na Suécia é relatada em $25 \%$, na Inglaterra $18 \%$ e na França $27,1 \%$. Em países orientais as cifras referidas são bem inferiores: Índia 7,5\%, Malásia $3 \%$ e China $0,8 \%{ }^{(10)}$.

A falta de dados epidemiológicos brasileiros e latinoamericanos, assinalada no I Consenso Brasileiro da DRGE,

${ }^{1}$ Departamento de Clínica Médica e ${ }^{2}$ Departamento de Medicina Social da ${ }^{3}$ Faculdade de Medicina da Universidade Federal de Pelotas, RS

Endereço para correspondência: Dr. Juvenal Soares Dias da Costa - Av. Duque de Caxias, 250 - 96030-002 - Pelotas, RS. e-mail: jsdc@ufpel.tche.br 
demanda observação nesse sentido, considerando-se as peculiaridades demográficas dessa região.

O objetivo do presente estudo foi a estimativa populacional da prevalência de pirose entre a população adulta na cidade de Pelotas, RS, Brasil.

\section{MATERIAL E MÉTODOS}

Realizou-se inquérito epidemiológico de base populacional para se estimar a prevalência de pirose na população adulta, a partir de 20 anos, residente na zona urbana da cidade de Pelotas, RS. O projeto foi aprovado pelo Comitê de Ética e Pesquisa da Faculdade de Medicina da Universidade Federal de Pelotas.

Estimou-se o tamanho da amostra, a partir da prevalência de 15\% descrita na literatura ${ }^{(5)}$, de erro amostral de $3 \%$ e nível de confiança de 95\%. O tamanho necessário para o processo de amostragem aleatória simples foi de 543 pessoas. Para a utilização do método de amostragem por conglomerados, corrigiu-se a estimativa e, assim, decidiu-se entrevistar 1.086 pessoas.

A inclusão dos indivíduos foi realizada através de visitas domiciliares em 40 setores censitários sorteados aleatoriamente. Em cada setor foram visitados 19 domicílios, totalizando 760 famílias. A partir de um quarteirão previamente sorteado, escolheu-se também de maneira aleatória o ponto de partida. Ao terminar a entrevista em determinado domicílio, sistematicamente, saltavam-se duas casas antes da próxima.

Ao final do trabalho de campo, encontraram-se 738 famílias. Portanto, 22 (2,9\%) famílias foram classificadas como perdas ou recusas.

Incluíram-se no estudo 1.263 pessoas entrevistadas entre as 1.372 encontradas a partir de 20 anos nos setores visitados. Dessa forma, as perdas e recusas individuais atingiram $7,7 \%$.

O trabalho de campo foi realizado entre março e setembro de 2001, com a aplicação de questionário padronizado e pré-codificado a todas as pessoas incluídas na pesquisa. Os entrevistadores, acadêmicos de Medicina, foram submetidos a programa de treinamento, realizando estudo piloto em outro setor não incluído na investigação, além de desconhecer os objetivos do deste.

Caracterizou-se como pirose ou azia a sensação de ardência ou queimação com início na região epigástrica e/ou retroesternal. Os entrevistadores foram instruídos a definir pirose ou azia como a sensação de ardência ou queimação que começa na região da "boca do estômago e sobe em direção à garganta". Quando julgado necessário, indicavam, com as mãos, as topografias de epigástrio e região retroesternal.

A pergunta foi realizada em relação à ocorrência do sintoma no último ano, no último mês e na última semana que precederam o momento da entrevista.
Foram também investigados alguns fatores associados à pirose, tais como piora com refeições ou com decúbito. As variáveis demográficas foram idade e sexo.

Realizou-se controle de qualidade aplicando-se questionários simplificados em $10 \%$ das pessoas incluídas na amostra.

A codificação e a entrada de dados através do programa Epi-Info foram realizadas duas vezes, com o intuito de diminuir os erros de consistência. A análise dos dados foi realizada através do programa SPSS, na qual foram feitas comparações entre a prevalência de pirose em relação às variáveis demográficas através das razões de prevalências, intervalos de confiança a $95 \%$ e do teste do qui-quadrado(2).

\section{RESULTADOS}

Entre as 1.263 pessoas incluídas no estudo, encontraram-se taxas de prevalências de pirose de 48,2\%, 32,2\% e 18,2\% para o último ano, mês e semana, respectivamente (Tabela 1). A prevalência de pirose no último ano, quando provocada pelas refeições, foi de $33 \%$, acentuada pelo decúbito $16,6 \%$ e associada aos dois fatores foi $12,1 \%$ (Tabela 2). Em relação à prevalência de pirose no último mês, encontraram-se percentuais de $23,0 \%, 11,4 \%$ e $8,5 \%$, provocada pelas refeições, acentuada pelo decúbito e associada aos dois fatores, respectivamente (Tabela 3 ).

O sintoma pirose na última semana foi observado em $14,3 \%$ associado com as refeições, 7,2\% relacionados ao decúbito e $6 \%$ quando analisados conjuntamente (Tabela 4).

TABELA 1 - Prevalência de pirose no último ano, mês e semana. Pelotas, RS, 2001

\begin{tabular}{lc}
\hline Variável & $\begin{array}{c}\text { Prevalência (intervalo de } \\
\text { confiança 95\%) }\end{array}$ \\
\hline Pirose no último ano & $48,2 \%(45,5$ a 51,0$)$ \\
Pirose no último mês & $32,2 \%(29,6$ a 34,8$)$ \\
Pirose na última semana & $18,2 \%(16,1$ a 20,3$)$ \\
\hline
\end{tabular}

TABELA 2 - Prevalência de pirose no último ano provocada por refeições, acentuada pelo decúbito e pela associação dos dois fatores. Pelotas, RS, 2001

\begin{tabular}{lc}
\hline Variável & $\begin{array}{c}\text { Prevalência (intervalo de } \\
\text { confiança 95\%) }\end{array}$ \\
\hline Pirose provocada por refeições & $33,0 \%(30,4$ a 35,6$)$ \\
Pirose acentuada pelo decúbito & $16,6 \%(14,6$ a 18,7$)$ \\
Pirose provocada por alimentos & $12,1 \%(10,3$ a 13,9$)$ \\
e acentuada pelo decúbito & \\
\hline
\end{tabular}


TABELA 3 - Prevalência de pirose no último mês provocada por refeições, acentuada pelo decúbito e pela associação dos dois fatores. Pelotas, RS, 2001

\begin{tabular}{lc}
\hline Variável & $\begin{array}{c}\text { Prevalência (intervalo de } \\
\text { confiança 95\%) }\end{array}$ \\
\hline Pirose provocada por refeições & $23,0 \%(20,6$ a 25,3$)$ \\
Pirose acentuada pelo decúbito & $11,4 \%(9,6$ a 13,2) \\
Pirose provocada por alimentos e & $8,5 \%(6,9$ a 10,0) \\
acentuada pelo decúbito & \\
\hline
\end{tabular}

TABELA 4 - Prevalência de pirose na última semana provocada por refeições, acentuada pelo decúbito e pela associação dos dois fatores. Pelotas, RS, 2001

\begin{tabular}{lc}
\hline Variável & $\begin{array}{c}\text { Prevalência (intervalo de } \\
\text { confiança 95\%) }\end{array}$ \\
\hline Pirose provocada por refeições & $14,3 \%(12,3$ a 16,2) \\
Pirose acentuada pelo decúbito & $7,2 \%(5,8$ a 8,6$)$ \\
Pirose provocada por alimentos e & $6,0 \%(4,7$ a 7,3$)$ \\
acentuada pelo decúbito & \\
\hline
\end{tabular}

Todas as formas do desfecho pirose estavam associadas com o sexo feminino, de maneira estatisticamente significativa. Contudo, não foi encontrada associação com relação à idade.

\section{DISCUSSÃO}

Um dos aspectos importantes em estudos populacionais é que a partir de seus achados, é possível realizar inferências e estimativas para toda a população. As apresentações dos intervalos de confiança permitem verificar as precisões das medidas ${ }^{(3)}$.

Do ponto de vista de delineamento, pode-se afirmar que o rigor metodológico do estudo pôde superar problemas inerentes ao seu desenho: viés de seleção e acaso ${ }^{(1)}$. Desta forma, verificou-se que as características demográficas dos indivíduos incluídos no estudo coincidem com a distribuição da população da cidade estudada. Afastouse a possibilidade da ocorrência de acaso em relação aos resultados encontrados, uma vez que os achados desta série são semelhantes a outros estudos populacionais, realizados em outros países.

Durante a revisão bibliográfica não foram encontrados estudos brasileiros de base populacional para prevalência de pirose com o rigor metodológico utilizado no presente trabalho. Estudos de prevalência baseados em dados de serviços cometem viés de seleção, uma vez que só incluem os indivíduos que procuram assistência médica e excluem indivíduos que apresentam o sintoma, porém não a procuram. O critério diagnóstico utilizado no presente estudo foi a ocorrência de pirose, no mínimo no último mês, provocada pelas refeições e acentuada pelo decúbito. O recordatório de um mês utilizado levou em consideração a possibilidade de que períodos de tempo maiores poderiam incorrer em viés de memória, conforme assinalado em outros estudos epidemiológicos $^{(6,12)}$. Outros estudos também se basearam em sintomas clínicos para definição de pirose $^{(9,11,15)}$.

Assim, a maior prevalência de pirose encontrada foi de $48,2 \%$ durante o ano precedente à entrevista, enquanto a menor foi de $6,0 \%$ correspondendo à semana que antecedeu a visita, provocada pelas refeições e acentuada pelo decúbito. A ocorrência mensal situou-se ao redor de $8,5 \%$. As prevalências foram semelhantes ou menores do que os outros estudos disponíveis, uma vez que no trabalho clássico de $\operatorname{DODDS}^{(5)}, 11 \%$ referiam pirose diária, $12 \%$ pirose semanal e $15 \%$ pirose mensal. Outros estudos ${ }^{(7,11)}$ estimam que, aproximadamente, $10 \%$ da população americana têm pirose diariamente e $40 \%$ relatam pirose mensalmente. Dados mais recentes apontam aumento da pirose de ocorrência semanal, 19,8\%(9) estimando-se que a prevalência na população adulta dos Estados Unidos seja de $44 \%{ }^{(14)}$.

Cabe ressaltar que a presença de pirose, bem como a sua freqüência, não é fator preditivo de lesão endoscópica. Aproximadamente 50\% dos pacientes com sintomas de DRGE, ou pHmetria ambulatorial de 24 horas anormal, indicando refluxo, apresentam aspecto normal da mucosa esofágica à endoscopia ${ }^{(6,15)}$. Portanto, o exame endoscópico mostrando mucosa esofagiana normal não exclui o diagnóstico de DRGE. A pirose é a manifestação clínica mais comum, conseqüente ao refluxo gastroesofágico, constituindo-se em excelente marcador para a doença. Contudo, deve-se ressaltar que, ao se utilizar esofagite endoscópica como padrão-ouro, o valor preditivo positivo da manifestação clínica poderá variar, de acordo com a prevalência da doença do refluxo, de $56 \%$ nos gastroenterologistas em hospitais a $25 \%$ na prática geral em populações não selecionadas ${ }^{(12)}$.

Sua alta prevalência deve chamar a atenção para aqueles indivíduos que possam estar expostos ao risco de complicações, bem como para aqueles que apresentem alterações da qualidade de vida.

\section{AGRADECIMENTO}

Ao Prof. Dr. Joaquim Prado Pinto de Moraes Filho por suas valiosas sugestões durante a elaboração deste artigo. 
Nader F, Costa JSD, Nader GA, Motta GLCL. Prevalence of heartburn in Pelotas, RS, Brasil: study in population base. Arq Gastroenterol $2003 ; 40(1): 31-34$

ABSTRACT - Objective - Estimation of the prevalence of heartburn in an adult population of Pelotas, RS, Brazil, once there is no national study done with adequate and specific statistic analysis. Design - Cross-sectional study in populational base. Method - Were included citizen over 20 years in samples obtained in 40 populational areas according to Brazilian Institute of Geography and Statistics. An uniform and precodified questionnaire was applied to all participants. A quality control was done using a simplified questionnaire in $10 \%$ of the people that were included in the sample. Codification and the input of the data was done two times through the Epi-Info programme in order to minimize the consistency errors. The analysis of the figures was done in the SPSS programme and obtained comparations between the prevalence of heartburn in relation to demographic variables through the prevalence rate, confidence interval of $95 \%$ and the ' $x$ - square test. Also were analysed the figures of heartburn associated to meals and decubit position. Results - Among 1,263 persons included in the study were found rates of the heartburn prevalence of $48.2 \%, 32.2 \%$ and $18.2 \%$ in the last year, month and week, respectively. All forms of heartburn were associated to females with statistic significance. There was no association with age. The prevalence of heartburn associated with meals and decubit position in the last year was $33 \%$ and $16.6 \%$, respectively. These association in the last month was $23 \%$ and $11.4 \%$ and in the last week was $14.3 \%$ and $7.2 \%$, respectively. Conclusions - The high prevalence found in this study should call attention to those persons which could be exposed to the risk of complications, as well as to those that have alterations in the life quality.

HEADINGS - Heartburn, epidemiology.

\section{REFERÊNCIAS BIBLIOGRÁFICAS}

1. Albhom A, Norell S. Introduction to modern epidemiology. Chestnut Hill: Epidemiology Resources; 1990

2. Altman DG. Practical statistics for medical research. London: Chapman \& Hall; 1997.

3. Andrade ALSS, Zicker F. Estudos de prevalência. Brasília: Organização PanAmericana da Saúde/Fundação Nacional de Saúde; 1997. p.33-42. (Métodos de investigação epidemiológica em doenças transmissíveis, v.1).

4. Dimenas E. Methodological aspects of evaluation of quality of life in upper gastrointestinal diseases. Scand J Gastroenterol 1993;28:18-21.

5. Dodds WJ. The pathogenesis of gastroesophageal reflux disease. AJR Am J Roentgenol 1988;151:49-56.

6. Fennerty MB, Sampliner RE. Gastroesophageal reflux disease and Barrett's esophagus. In: DiMarino AJ, Benjamin SB, editors. Gastrointestinal disease an endoscopic approach. Malden, Mass.: Blackwell Science; c1997. p.130-46.

7. A Gallup Survey on Heartburn Across America. Princeton, NJ: The Gallup Organization; 1968.

8. Kroeger A. Health interview surveys in developing countries: review of the methods and results. Int J Epidemiol 1983;12:465-81.

9. Locke GR, Talley NJ, Fett SL, Zinsmeister AR, Melton LJ. Prevalence and clinical spectrum of gastroesophageal reflux: a population-based study in Olmsted County, Minnesota. Gastroenterology 1997;112:1448-56.
10. Moraes-Filho JPP, Cecconello I, Gama-Rodrigues J, Castro LP, Henry MA, Meneghelli UG, Quigley E. Brazilian consensus on gastroesophageal reflux disease: proposals for assessment, classification and management. Am J Gastroenterol 2002;97:241-8.

11. Nebel OT, Michael F, Fornes MC, Castel DOC. Symptomatic gastroesophageal reflux: incidence and precipitating factors. Am J Dig Dis 1976;21:953-6.

12. Petersen $\mathbf{H}$. The prevalence of gastro-oesophageal reflux disease. Scand $\mathbf{J}$ Gastroenterol 1995;30 (Suppl 211):5-6.

13. Ross DA, Vaughan JP. Health surveys in developing countries: a methodological review with recommendations for future surveys. London: School of Hygiene and Tropical Medicine; 1984. (EPC Publications 4).

14. Roy AM, Orlando RC. Gastroesophageal reflux disease. Curr Opinion Gastroenterol 2001;17:359-65.

15. Winters C Jr, Spurling TJ, Chobanian SJ, Curtis DJ, Esposito RL, Hacker 3d JF, Johnson DA, Cruess DF, Cotelingan JD, Gurney MS. Barrett's esophagus. A prevalent, occult complication of gastroesophageal reflux disease. Gastroenterology 1987;92:118-24. 